\title{
Substitution of aromatic residues in the macrocyclic opioid peptide [D-Trp]CJ-15,208 alters the opioid activity profile in vivo
}

Jane Aldrich ${ }^{1}$, Dmitry Yakovlev ${ }^{1}$, Nicolette Ross ${ }^{2}$, Shainnel Eans ${ }^{1}$, Heather Stacy $^{1}$, Thomas Murray ${ }^{3}$, Jay McLaughlin ${ }^{1}$

${ }^{1}$ University of Florida, United States
${ }^{2}$ University of Kansas, United States
${ }^{3}$ Creighton University, United States

https://doi.org/10.17952/35EPS.2018.061

Kappa opioid receptors (KOR) are involved in responses to stress, and KOR antagonists have potential therapeutic application for the treatment of substance abuse and mood disorders.[1] The macrocyclic peptide natural product CJ-15,208 was reported to be a KOR antagonist in vitro,[2] but the stereochemistry of the tryptophan residue was not reported. Therefore we synthesized both the L- and D-Trp isomers of CJ-15,208 [3] and evaluated their opioid activity profile in vitro and in vivo.[4] In vivo [D-Trp]CJ-15,208 antagonizes KOR after both central (intracerebroventricular, i.c.v.) [4] and oral [5] administration. It can penetrate the blood-brain barrier after oral administration to antagonize central KOR and prevent stressinduces reinstatement of extinguished cocaine-seeking behavior.[5] Therefore [D-Trp]CJ-15,208 is a promising lead peptide for structuralmodification to enhance its in vivo activity after oral administration.

Initially the alanine analogs of [D-Trp]CJ-15,208 were prepared and evaluated both in vitro and in vivo.[6] Unexpectedly all of the alanine analogs exhibited potent full agonist (antinociceptive) activity in vivo in the mouse $55^{\circ} \mathrm{C}$ warm-water tail withdrawal assay, in contrast to the parent peptide that exhibits only modest antinociception ( $40 \%$ of the maximum response) at the highest dose tested ( $30 \mathrm{nmol}$ i.c.v.). Therefore subsequent modifications focused on conservative modifications to the aromatic residues, including modifications that may decrease or prevent metabolism by liver oxidative enzymes.

\section{Methods}

The peptides were synthesized by a combination of solid phase peptide synthesis of the linear precursors as previously described, followed by cyclization in solution and purification by normal phase column chromatography.[3,7]

The peptides were evaluated in vitro and in vivo using methods described previously.[6] Opioid receptor affinity was determined in radioligand binding assays using cloned receptors. [8] The peptides were evaluated in vivo for agonist (antinociceptive) and antagonist activity in C57BL/6 mice in the $55{ }^{\circ} \mathrm{C}$ warm-water tail withdrawal assay. To determine opioid receptor involvement in observed agonist activity the antinociception was measured in KOR and mu opioid receptor (MOR) knockout mice, as well as in wild-type mice pretreated with the delta opioid receptor antagonist naltrindole $(0.5 \mathrm{mg} / \mathrm{kg}$ i.p.). To determine KOR antagonist activity mice were pretreated with peptide prior to the administration of the KOR selective agonist U50,488 (10 mg/kg, i.p.), and antinociception measured 40 min later.

[D-Trp]CJ-15,208, $\left[\mathrm{Ala}^{1}, \mathrm{D}-\mathrm{Trp}\right] \mathrm{CJ}-15,208$ and nor-BNI were evaluated for their ability to prevent reinstatement of extinguished morphine conditioned place preference (CPP) using procedures similar to those described previously for evaluation of compounds for their ability to prevent reinstatement of extinguished cocaine CPP. [6] Mice were subjected to 4 days of place conditioning in a counterbalanced morphine CPP paradigm. The mice were then evaluated for their place preference twice weekly until extinction was established, which required 3-6 weeks. Following extinction mice were pretreated with either vehicle or peptide and subsequently subjected to forced swim stress for two days as previously described.[4] Mice were tested for place preference on the day after stress exposure. The results are presented as the difference in the time spent in the morphine-paired vs. vehicle-paired compartments.

\section{Results}

Substitutions on the phenyl ring of $\mathrm{Phe}^{3}$ in [D-Trp]CJ-15,208 (Figure 1) can alter the in vivo opioid activity profile of the resulting analogs. The effects of incorporation of a fluorine onto this ring depended on the position of this heteroatom on the ring. The m-fluoro-substituted analog retained KOR antagonism, but also exhibited 
antinociception following i.c.v. administration $\left(\mathrm{ED}_{50}(95 \%\right.$ confidence interval $)=33(12-102)$ nmol, Figure 2); evaluation in knockout mice indicated that both MOR and KOR are involved in the observed antinociception. In contrast, the para-substituted analog exhibited minimal antinociception $(<35 \%)$ and weak KOR antagonist activity only at the highest dose tested (100 nmol i.c.v.). These results are consistent with the KOR affinities of the peptides ( $\mathrm{Ki}=43 \pm 12$ vs. $134 \pm 63 \mathrm{nM}$ for the meta and para substituted analogs, respectively). Other substitutions for $\mathrm{Phe}^{3}$ also resulted in mixed agonist/KOR antagonist activity, although the receptors involved in the antinociception varied with the different analogs. However, some substitutions (e.g. His in place of Phe ${ }^{3}$ ) resulted in analogs that produced antinociception but lost KOR antagonist activity.<smiles>O=C(NC(Cc1ccccc1)Cc1ccccc1)[C@H](Cc1c[nH]c2ccccc12)NC(=O)[C@H](Cc1ccccc1)NC(=O)[C@@H]1CCCN1C(=O)C1CCCC1</smiles>

Figure 1: Structure of [D-Trp]CJ-15,208 with numbering of Phe residues.

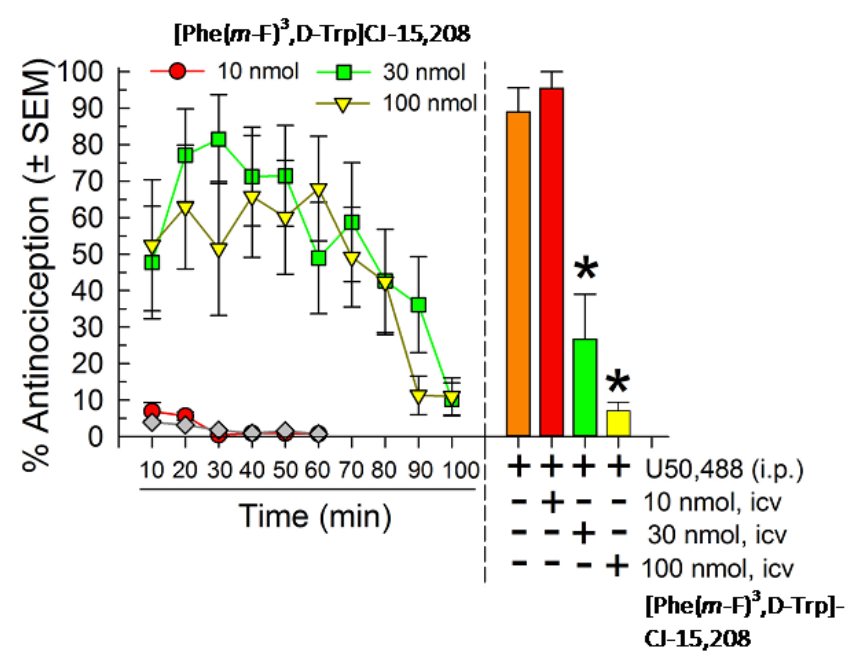

Figure 2: Antinociceptive and KOR antagonist activity of [Phe(m-F)3,D-Trp]CJ-15,208 in the mouse 55 oC warm-water tail assay. * significantly different $(p<0.05)$ from U50,488 alone.

KOR antagonists can prevent reinstatement of extinguished cocaine-seeking behavior,[1] and a "functional KOR antagonist" (buprenorphine plus naltrexone) significantly improved drug abstinence in heroin-dependent patients compared to patients treated with naltrexone alone.[9] Therefore we examined the ability of [DTrp]CJ-15,208, the alanine analog $\left[\mathrm{Ala}^{1}, \mathrm{D}-\mathrm{Trp}\right] \mathrm{CJ}-15,208$, which also exhibits KOR antagonism and prevents stress-induced reinstatement of extinguished cocaine seeking behavior,[6] and the small molecule KOR antagonist nor-BNI to prevent stress-induced reinstatement of morphine seeking behavior (Figure 3). Pretreatment with all three of the compounds significantly decreased the reinstatement of morphine seeking behavior in the conditioned place preference assay. Other analogs of [D-Trp]CJ-15,208 that exhibit KOR antagonism also prevented stress-induced reinstatement of morphine CCP, demonstrating the potential of these macrocyclic peptides in the treatmentof drug abuse. 


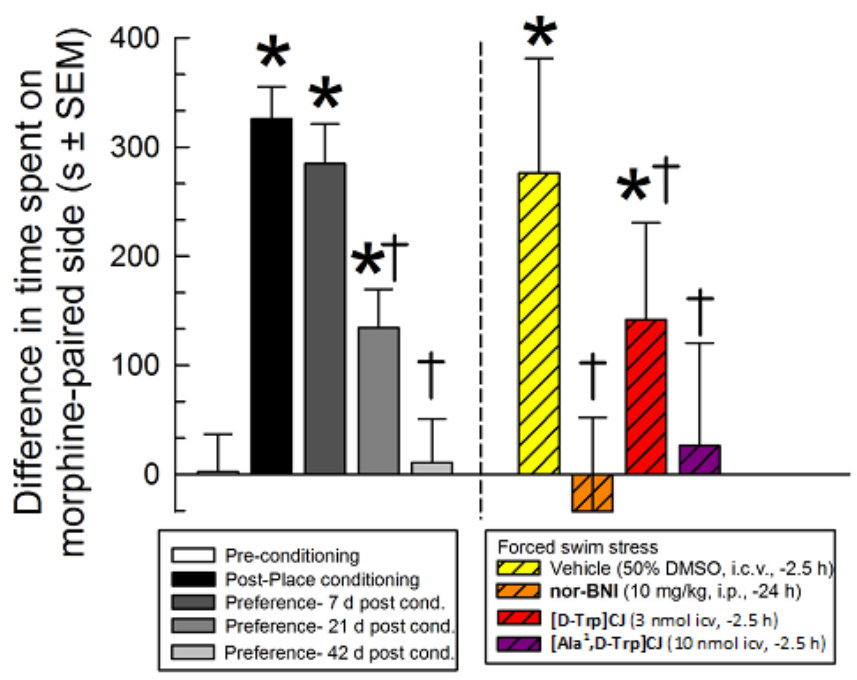

Figure 3: [D-Trp]CJ-15,208, [Ala1,D-Trp]CJ-15,208 and nor-BNI prevented reinstatement of stress-induced morphine conditioned place preference. Following conditioning with morphine, extinction occurred over the next 6 weeks (left bars). Exposure to forced swim stress reinstated morphine CPP (yellow bar). Pretreatment with nor-BNI (10 mg/kg i.p., orange bar), [D-Trp]CJ-15,208 ([D-Trp]CJ, 3 nmol i.c.v., red bar) or [Alal,DTrp]CJ-15,208 ([Alal,D-Trp]CJ, 10 nmol i.c.v., purple bar) significantly decreased reinstatement of CPP. * and $\dagger$, significantly different from preconditioning and post-conditioning, respectively.

In conclusion, substitutions for $\mathrm{Phe}^{3}$ in [D-Trp]CJ-15,208 can alter the opioid activity profile of the resulting peptides. Most of the analogs retained KOR antagonism, with several also exhibiting antinociception that was mediated by multiple opioid receptors. Consistent with their KOR antagonism [D-Trp]CJ-15,208 and selected analogs also prevented stress-induced reinstatement of morphine-seeking behavior. Further studies are ongoing in our laboratories to further characterize and develop these promising macrocyclic peptides.

\section{Acknowledgements}

This research was supportedby grants PR141230 and PR141230P1 from the U.S. Army and grant R01 DA018332 from the National Institute on Drug Abuse.

\section{References}

1. J. V. Aldrich and J. P. McLaughlin, AAPS J., 2009, 11, 312-322.

2. T. Saito, H. Hirai, Y.-J. Kim, Y. Kojima, Y. Matsunaga, H. Nishida, T. Sakakibara, O. Suga, T. Sujaku and N. Kojima, J. Antibiot., 2002, 55, 847-854.

3. N. C. Ross, S. S. Kulkarni, J. P. McLaughlin and J. V. Aldrich, TetrahedronLett., 2010, 51, 5020-5023.

4. N. C. Ross, K. J. Reilley, T. F. Murray, J. V. Aldrich and J. P. McLaughlin, Br. J. Pharmacol., 2012, 165, 1097-1108.

5. S. O. Eans, M. L. Ganno, K. J. Reilley, K. A. Patkar, S. N. Senadheera, J. V. Aldrich and J. P. McLaughlin, Br. J. Pharmacol., 2013, 169, 426-436.

6. J. V. Aldrich, S. N. Senadheera, N. C. Ross, K. J. Reilley, M. L. Ganno, S. O. Eans, T. F. Murray and J. P. McLaughlin, Br. J. Pharmacol., 2014, 171, 3212-3222.

7. S. N. Senadheera, S. S. Kulkarni, J. P. McLaughlin and J. V. Aldrich, in Peptides: Building Bridges, ed. M. Lebl, American Peptide Society, San Diego, CA, 2011, pp. 346-347.

8. S. Arttamangkul, J. E. Ishmael, T. F. Murray, D. K. Grandy, G. E. DeLander, B. L. Kieffer and J. V. Aldrich, J. Med. Chem., 1997, 40, 1211-1218.

9. G. Gerra, A. Fantoma and A. Zaimovic, J. Psychopharmacol., 2006, 20, 806-814. 